\title{
Referral of Facial Nerve Palsy to Specialist Care According to NICE (2012) Guidelines
}

Rowe. $A^{1}$, George. $S^{2} \&$ Harper-Machin. $A^{3}$

1. University of Liverpool 2. St. Helens and Knowsley Teaching Hospitals NHS Trust 3. St. Helens and Knowsley Teaching Hospitals NHS Trust

\section{Introduction:}

- Facial nerve palsy (FNP) is a serious problem that requires timely referral in order to prevent serious psychological sequalae.

- The UK annual incidence of FNP is up to 37.7 per 100,000 people, occurring in those aged 15-60, diabetics and pregnant women $(1,2)$.

- $29 \%$ of people with FNP will not recover normal facial nerve function and live with residual paralysis (defined in this study as lasting $>6$ months) (3).

- NICE (2012) guidance recommends referral to a plastic surgeon (with a special interest in facial reconstruction) if there is residual paralysis after 6-9 months (4).

Aim:

- Primary outcome: to determine how many people with an unresolved FNP are referred to a facial reconstructive plastic surgeon within 6-9 months.

- Secondary outcomes: aetiology of residual FNP and how many FNP cases are referred to specialists other than plastic surgery.

\section{Methods:}

- We performed a retrospective audit of all patients presenting with FNP to the trust between April 2016 and March 2017. Data was obtained from electronic patient records and operation notes.

\section{Results:}

- $\mathrm{N}=85$ patients were identified, $\mathrm{N}=40$ excluded (for not being referred to a specialist) giving a final sample of $\mathrm{N}=45.22 / 45$ patients had a residual FNP.

- 33 were female $(73 \%)$ and 12 were male (27\%) (M:F ratio 1:2.75).

- The average age was 60 (Median age 66).

- Plastic surgery was the most common specialty referral regardless of FNP duration (20/41)

- This was excluding recurrent FNP and one patient who was referred to an unknown specialty

- The other specialties referred to can be found in Chart 1.

\section{Chart 1: Specialist Referral $(n=41)$}

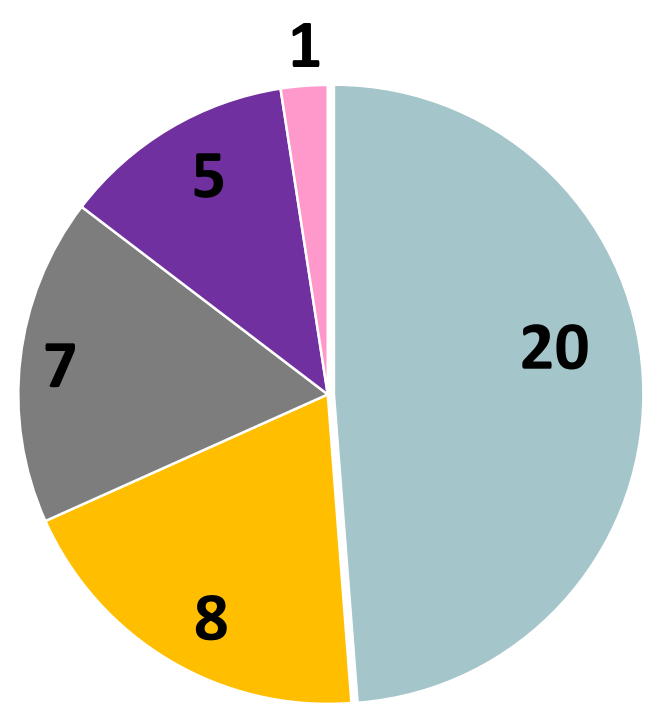

\footnotetext{
Plastics (49\%)
}

ENT (20\%)

Opthalmology (17\%)
- Median time from diagnosis to specialist referral was 6 years ( $N=21,1$ not documented)

- $18 / 22$ patients with a residual FNP were referred plastic surgery. Of these, only four (18\%) were referred within 6-9 months.

Reasons for why they were not referred as per guidelines can be found in Chart 2.

\section{Chart 2: Reasons for Late Referral $(n=14)$}

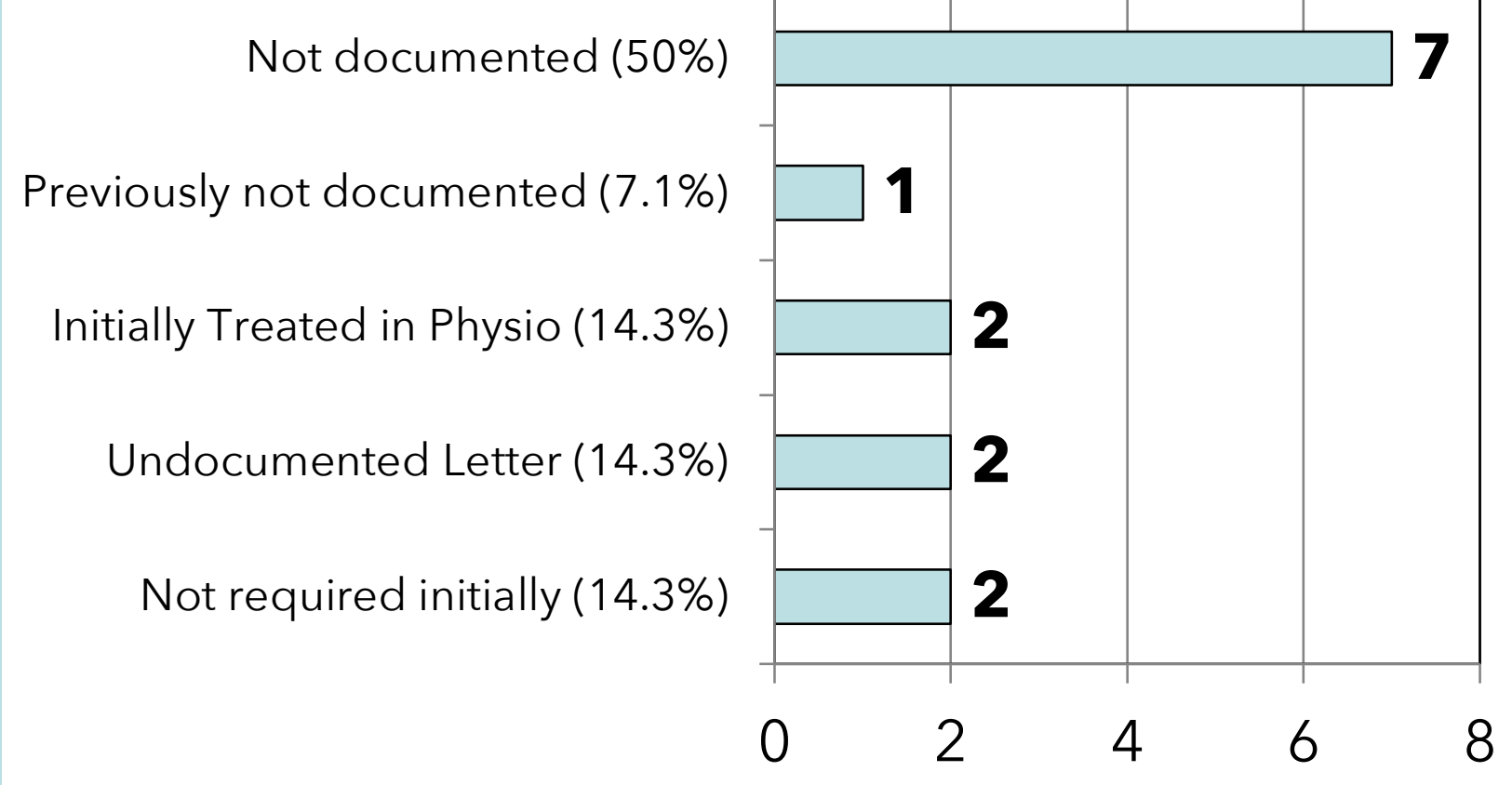

Post-operative complications were the main cause of residual FNP 12/22 (55\%- see Chart 3), followed by Bell's palsy, 7 $(32 \%)$, Ramsay hunt syndrome, $2(9 \%)$ and congenital, 1 $(4 \%)$.

\section{Chart 3: Sub-group analysis of post- operative complication aetiology}

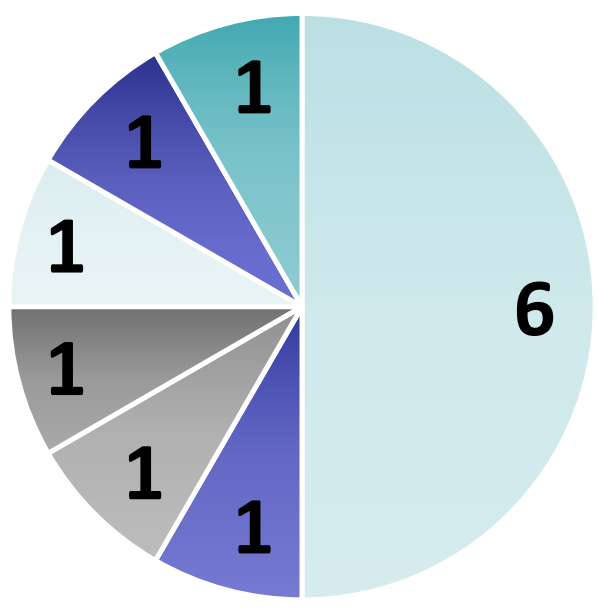

Acoustic neuroma (50\%)

- Carotid malignancy (8.3\%)

Metastatic pre-auricular SCC

(8.3\%)

- Post-auricular SCC (8.3\%)

Brain stem cavernoma $(8.3 \%)$

- Malignant melanoma (8.3\%)

\section{Conclusion:}

- $82 \%$ of patients with a residual FNP were not referred to a specialist plastic surgeon within 6-9 months.

- Most referrals for FNP were made to plastic surgery, followed by ENT, ophthalmology and neurology.

- The most common cause of residual FNP were post-operative complications. (50\% from acoustic neuroma resections).

\section{Raise awareness:}

\section{Way Forward:}

- Contact primary care physicians to make clear the service provided by the trust and current referral guidelines for FNP.

\section{Review of NICE guidance:}

- Reduce the point of referral $<6$ months since treatment is timedependent, with limited treatment options at 12 months.

- We found in our study that most patients referred were approaching 12 months of having a residual FNP.

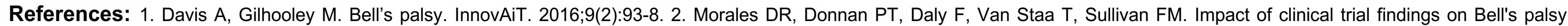

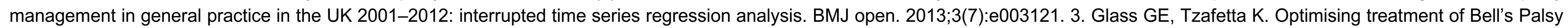

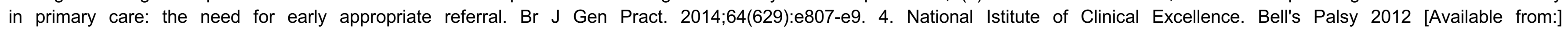
https://cks.nice.org.uk/bells-palsy\#!scenario (Accessed 18 ${ }^{\text {th }}$ March 2018) 